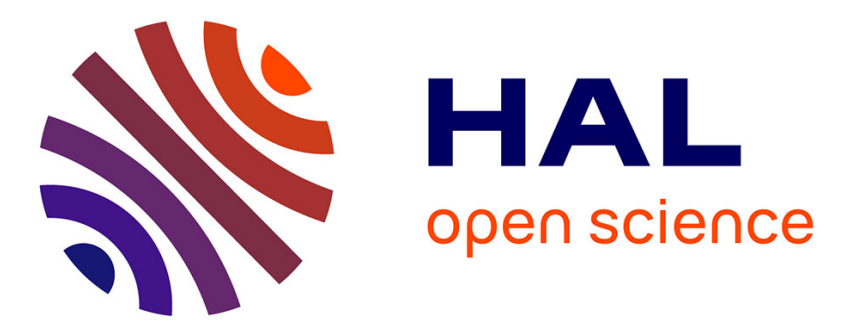

\title{
Toward accurate segmentation of the LV myocardium and chamber for volumes estimation in gated SPECT sequences
}

Diane Lingrand, Arnaud Charnoz, Malik Koulibaly, Jacques Darcourt, Johan Montagnat

\section{To cite this version:}

Diane Lingrand, Arnaud Charnoz, Malik Koulibaly, Jacques Darcourt, Johan Montagnat. Toward accurate segmentation of the LV myocardium and chamber for volumes estimation in gated SPECT sequences. European Conference on Computer Vision (ECCV'04), May 2004, Prague, Czech Republic. pp.1-10. hal-00691648

\section{HAL Id: hal-00691648 https://hal.science/hal-00691648}

Submitted on 26 Apr 2012

HAL is a multi-disciplinary open access archive for the deposit and dissemination of scientific research documents, whether they are published or not. The documents may come from teaching and research institutions in France or abroad, or from public or private research centers.
L'archive ouverte pluridisciplinaire HAL, est destinée au dépôt et à la diffusion de documents scientifiques de niveau recherche, publiés ou non, émanant des établissements d'enseignement et de recherche français ou étrangers, des laboratoires publics ou privés. 


\title{
Toward accurate segmentation of the LV myocardium and chamber for volumes estimation in gated SPECT sequences
}

\author{
Diane Lingrand ${ }^{1}$, Arnaud Charnoz ${ }^{1}$, Pierre Malick Koulibaly ${ }^{2}$, Jacques \\ Darcourt $^{2}$, and Johan Montagnat ${ }^{3}$ \\ 1 I3S, UNSA/CNRS UMR 6070 - B.P. 121 - F06903 Sophia Antipolis - FRANCE \\ lingrand@i3s.unice.fr \\ http://www.i3s.unice.fr/ lingrand/ \\ 2 Centre Antoine Lacassagne - Nice - FRANCE \\ 3 CREATIS, CNRS/INSA Lyon - F69621 Villeurbanne Cédex - FRANCE \\ johan@creatis.insa-lyon.fr
}

\begin{abstract}
The left ventricle myocardium and chamber segmentation in gated SPECT images is a challenging problem. Segmentation is however the first step to geometry reconstruction and quantitative measurements needed for clinical parameters extraction from the images. New algorithms for segmenting the heart left ventricle myocardium and chamber are proposed. The accuracy of the volumes measured from the geometrical models used for segmentation is evaluated using simulated images. The error on the computed ejection fraction is low enough for diagnosis assistance. Experiments on real images are shown.
\end{abstract}

\section{Introduction}

The Left Ventricle (LV) myocardium accurate segmentation in gated SPECT (Single Photon Emission Computed Tomography) images is a challenging problem due to the high level of noise and the signal drops resulting of insufficiently perfused regions. The LV chamber automated segmentation is even more difficult as the upper bound of the ventricle does not appear in the images. However, the accurate segmentation of the LV myocardium and chamber is very important for the estimation of the heart wall thickness and the chamber volume variation during the heart cycle. These parameters are needed to estimate clinically well established diagnosis indicators such as the ejection fraction.

In this paper, we propose an implicit model-based segmentation algorithm of the LV myocardium and chamber. Our model is guided by the need of accuracy for volumes quantitative estimation. Indeed, the coarse spatial and temporal resolution of gated SPECT images causes large partial volume effects that can significantly alter the volume estimation results. This paper follows an earlier study on levelset-based segmentation of gated SPECT image [4].

To model objects and segment images, both explicit [20] and implicit [16,2] deformable models have been proposed in the literature [15]. The levelset has 
been widely used in segmentation $[17,3,5]$, medical images segmentation $[13,18$, $8,11,14]$, heart segmentation [9] and SPECT images segmentation [7]. Some are taken into account shape priors [6]. Contrarily to many earlier approaches, our algorithm is taking into account a complete heart cycle sequence rather than processing volume frames independently. It is therefore better able to filter the image noise and to take into account temporal partial volume effects.

\section{Segmentation Model}

\subsection{LV myocardium model}

The LV myocardium is modeled using a levelset-based method. The levelset provides a geometrical representation of the LV as well as a deformation process needed for extracting the myocardium shape from the image. In the levelset framework, a surface model $S$ is implicitly represented as the 0 isosurface of a higher dimension function $u$. $S$ deforms when $u$ evolves according to an evolutive equation. Most evolution criteria found in the literature are spatial [12]. In the case of dynamic sequences, we prefer the Debreuve et al criterion [7]:

$$
\frac{\partial u_{n}}{\partial t}=\left(\lambda_{\text {in }}\left(I_{n}-\mu_{i n_{n}}\right)^{2}-\lambda_{\text {out }}\left(B-I_{n}\right)^{2}+\lambda_{c} \kappa_{n}\right)\left\|\nabla u_{n}\right\|
$$

where $I_{n}$ represents the image at instant $n$. The whole sequence is used in order to filter noise and determine the mean background intensity $B$, reestimated at each iteration. $\kappa_{n}$ is the curvature at instant $n$ and $\mu_{i n_{n}}$ the mean of image $n$ internal part, also reestimated at each iteration from the zero level of $u_{n} \cdot \lambda_{i n}$, $\lambda_{\text {out }}$, and $\lambda_{c}$ are weight parameters. This criterion makes the hypothesis that the image is composed of a uniform intensity region (the object to segment) and a background $B$. This approximation is only roughly valid for SPECT images due to the image noise, the inhomogeneity of the heart and the perfusion defaults causing signal drops. A forward Euler based on finite differences is used for PDE resolution. We note that there is no relation between iteration time $(\mathrm{t})$ and physical time (n).

\subsection{LV chamber model}

The LV chamber surface is delimited by the myocardium inner boundaries and the valves plane on top. However, the LV myocardium has a U shape opened on top in gated SPECT images and the valves are not visible. A method to enclose the chamber volume is therefore needed.

User guided methods. Some manual or semi-manual methods have been proposed in the literature. In [7], the authors manually set the two planes location. The result of this method is very user dependent. Faber, Cooke et al [10] approximate the LV valves by two fixed planes (see left of figure 1). The location and orientation of the two planes were empirically fixed on a dataset and is merely 
valid for a given acquisition protocol. Moreover, it is difficult to ensure that the chamber volume is always closed by the two planes: the myocardium upper part is irregular and holes are likely to appear between the myocardium boundaries and the planes.

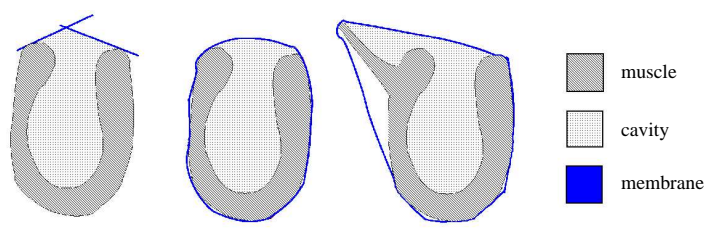

Fig. 1. Left: manual LV chamber closure. Center: membrane algorithm. Right: segmentation error inducing a volume estimation error.

Membrane algorithm To face the difficulty to accurately close the ventricle using planes, a new convex envelope algorithm was developed. This membrane method, depicted in center of figure 1 , is completely automatic. The membrane is a deformable surface initialized from the result of the myocardium segmentation and deformed using the following evolution equation:

$$
\frac{\partial u}{\partial t}=\left(\lambda_{1} I^{2}+\lambda_{2} \kappa\right)\|\nabla u\|
$$

where $I$ is a binary image resulting from the myocardium segmentation. The drawback of the membrane method is its sensitivity to the correct LV segmentation: for example when the visible bright region shape is not a U-shape. The membrane encloses the outliers and the inside volume is poorly estimated as illustrated in right of figure 1.

Once the membrane has been deformed, the LV chamber is obtained by binary image processing: a binary myocardium image is produced from the myocardium segmentation and the chamber is filled up to the membrane boundary. An isosurface of the resulting inner volume is computed as illustrated in figure 9. From the LV chamber volume, we can estimate the heart ejection fraction (EF). The EF is computed as the ratio between the volume of blood ejected at each heart beat (the difference of volumes between the chamber at end of dilation phase, or diastole, and contraction phase, or systole) over the chamber maximal volume: $E F=\left(V_{d}-V_{s}\right) / V_{d} \times 100 \%$ where $V_{d}$ and $V_{s}$ are the end of diastole and end of systole volumes, respectively.

\subsection{Challenging the homogeneous intensity region hypothesis}

The criterion 1 used in this study is based on the hypothesis that the image is composed of an homogeneous object on an homogeneous background. This is 
only roughly true in real images due to two partial volume effects and temporal blurring.

Partial volume effects. The partial volume effect is responsible for myocardium intensity variations during the cardiac cycle: the myocardium appears brighter at end of systole and darker at end of diastole. When the thickness of the myocardium is only a few voxels wide (at the end of diastole), many voxels do not contain only myocardium but also part of the outside region, lowering their intensity. Conversely, at end of systole, the thickening of the muscle leads to brighter muscle voxels. This artefact is used as an index of wall thickenning [1]. We can observe this phenomena on figure 2 .

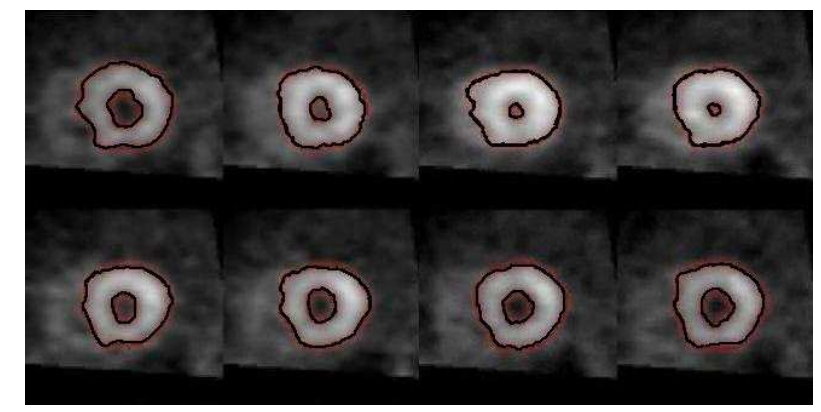

Fig. 2. Time frames 0 to 7 , from left to right and top to bottom, showing one short axis slice. The intensity is higher in frames 2 and 3 (end of systole) than in the others.

Temporal blurring. Due to the images reconstruction process, a blurring appears in the image sequences. This temporal blurring is mostly visible at the base (top) of the myocardium while the more static apex part is unaffected. The visual consequences are that (i) during diastole, extremities of the ventricle muscle are darker than the apex and (ii) during systole, borders of the muscle near the apex are darker.

Consequences of segmentation errors on volume estimation. Partial volume effects and temporal blurring combine their effects, leading to different segmentation errors during the systole and diastole phases. At end of diastole, the myocardium extremities are darker and tend to be truncated. The temporal blurring, will also cause the myocardium to appear slightly thicker than it is in reality. This leads to underestimating the chamber volume. Conversely at end of systole, the myocardium extremities are overestimated while the myocardium appears slightly thinner than it should be. This leads to overestimating the chamber volume. The EF estimation is significantly affected by the combination of these segmentation errors. Figure 4 shows the erroneous estimated volumes using the segmentation algorithm for different weights of the internal force term 
$\lambda_{i n}$. Although they appear visually insignificant, these segmentation errors have a drastic impact on the volume estimations. Due to the coarse resolution of gated SPECT images and the small size of the LV, even an error of only one voxel all along the LV chamber surface leads to an error of about $50 \%$ of the chamber volume, making the computed $\mathrm{EF}$ absolutely meaningless.

\section{Segmentation and quantification experiments}

To validate the algorithm accuracy, experiments on simulated images were first performed. With simulated images, a ground truth (the actual volumes of the virtual objects used for simulation) is known and the algorithm can be quantitatively evaluated. Experiments were then lead on real images for which no ground truth is available.

\subsection{Experiments on simulated images}

Simulating images using the NCAT phantom. W.P. Segars [19] has developed a four-dimensional NURBS-based CArdiac-Torso (NCAT) phantom for simulating nuclear medicine images. The organ models are based on non-uniform rational B-splines which define continuous surfaces. The phantom can thus be used at any spatial resolution. An important innovation is the extension of NURBS to a fourth dimension, time, to model the cardiac beat and the respiratory motion. Given a model of the physics of the nuclear imaging process, simulated images of the numerical phantom can be computed by the NCAT simulator. The main advantage of using computerized organ models in medical studies is that the exact anatomy and physiological functions of the phantom are known, thus providing a gold standard against which the image processing and reconstruction algorithms can be evaluated quantitatively.

Volume estimation. Figure 3 shows an example of volume estimation after segmentation of an image produced by the NCAT simulator and extraction of the chamber by the membrane algorithm. The volume estimation error is small compared to the spatial resolution of the simulated images (less than $6 \%$ in the worst case) and the error on the computed EF is lower than $2 \%$. The membrane algorithm therefore estimates a realistic closure of the LV boundary.

Realistic images. Raw images produced by the NCAT simulator are not realistic since they are not noisy and they do not introduce temporal blurring as described in section 2.3. For further evaluating our algorithm, an artificial temporal blurring was introduced by convolving the longitudinal sequences with a Gaussian kernel in the time direction $(\sigma=6)$, and a spatial Gaussian noise $(\sigma=4)$ was added in each image. Straight segmentation of blurred and noisy images is not satisfying. A high level of noise requires increasing the internal 


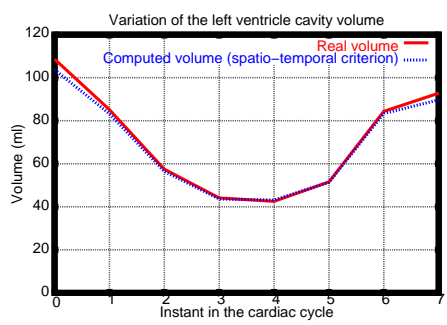

Fig. 3. Comparison of the LV chamber volume estimation on simulated NCAT images against the ground truth.

force weight. However, this also causes less precise location of the myocardium boundaries.

Different internal weight values have been tested in the criterion 1. Fixing $\lambda_{\text {out }}=1$ and $\lambda_{c}=1$, figure 5 shows the segmentation results for different values of $\lambda_{\text {in }}$. For low values of the internal force weight $\left(\lambda_{\text {in }}=1\right.$ and $\left.\lambda_{\text {in }}=2\right)$, the region near the apex is poorly segmented: the myocardium surface is too thick. For a higher value $\left(\lambda_{\text {in }}=3\right)$, the thickness is correct but a significant part of the extremities is truncated. A small part of the extremities is also truncated for $\lambda_{\text {in }}=1$, due to the temporal blurring (for better visualization, we superimposed the segmentation results on the original NCAT images but the segmentation is computed on blurred and noisy images). Figure 4 shows that the estimated volume of the LV chamber is indeed under-evaluated except at the end of systole. Both myocardium extremities troncature (for high values of $\lambda_{i n}$ ) and myocardium thickness overestimates (for low values of $\lambda_{i n}$ ) lead to underestimating the chamber volume.

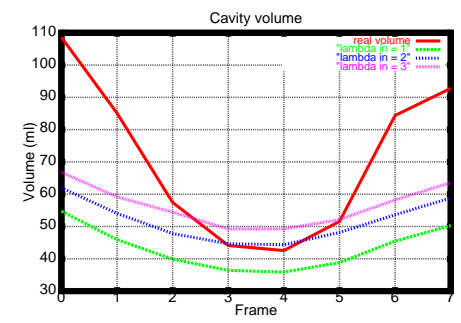

Fig. 4. LV chamber volume after segmentation of NCAT simulated data for different values of $\lambda_{\text {in }}$ and ground truth.

\subsection{A new adaptive algorithm}

Since the accurate segmentation of the different parts of the myocardium requires different tunings of the relative weights of the internal and external objects and 


$$
\lambda_{i n}=1
$$

$\lambda_{i n}=2$

$\lambda_{\text {in }}=3$
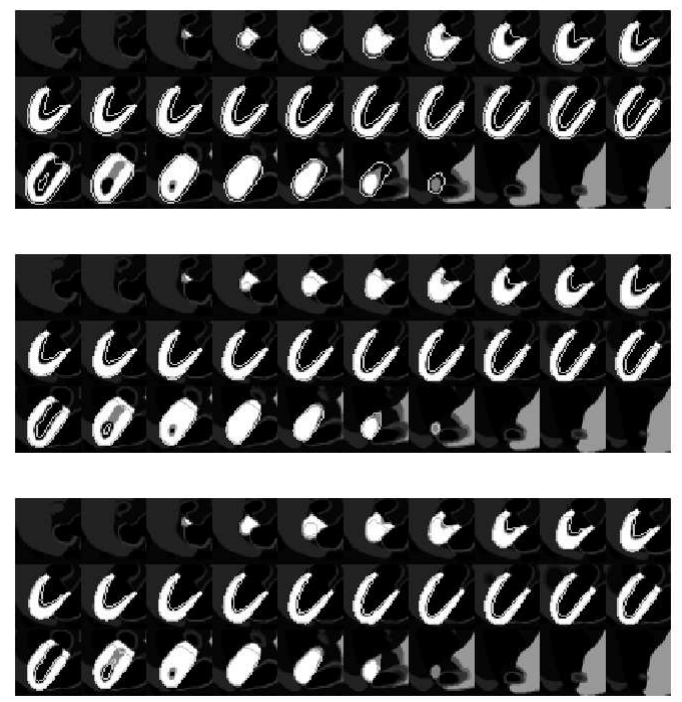

Fig. 5. Segmentation results for $\lambda_{\text {in }}=1$ (top), $\lambda_{\text {in }}=2$ (center), and $\lambda_{\text {in }}=3$ (bottom).

no satisfying trade-off can be found for the complete image, we propose an adaptive algorithm described by the following steps:

- Normal segmentation of the end of systole volume. The temporal blurring is minimum and the myocardium intensity is maximum at end of systole.

- LV barycenter and principal axis estimation. The principal axis computed from the segmented image roughly corresponds to the heart long axis.

- Image volume splitting. The volume space is split by several short axis planes. Different parameters can be attributed to each space region.

Locating the heart long axis. The end of systole volume presents the lowest temporal blurring and the highest myocardium contrast. It is therefore the easiest frame to segment. Moreover, this stage is not very sensitive to small segmentation errors. The myocardium is first extracted in this frame. The resulting model is used to produce a binary image. Only the largest connex component is kept from this image to remove outliers. The LV barycenter $(\bar{x}, \bar{y})$ and the principal axis are estimated. The principal axis is the eigenvector corresponding to the highest eigenvalue of the inertia matrix:

$$
M=\left[\begin{array}{ll}
m_{20} & m_{11} \\
m_{11} & m_{02}
\end{array}\right] \text { with } m_{i j}=\sum(x-\bar{x})^{i}(y-\bar{y})^{j} \delta(x, y)
$$

Estimation of the different planes. The image volume is split by planes normal to the heart long axis estimated in the previous step. The first volume region 
contains the heart apex. This region is delimited by a plane orthogonal to the principal axis and close enough to the LV barycenter to cut the myocardium extremities segmented at the end of systole with $\lambda_{i n}=1$ (see figure 6). The last region will fall outside the ventricle, beyond the myocardium extremities. It is determined by a plane parallel to the first one, and outside the segmentation obtained at the end of diastole with $\lambda_{i n}=3$. Two other planes, equally spaced between the two previous ones, finish to split the image volume in 5 regions (see figure 6).

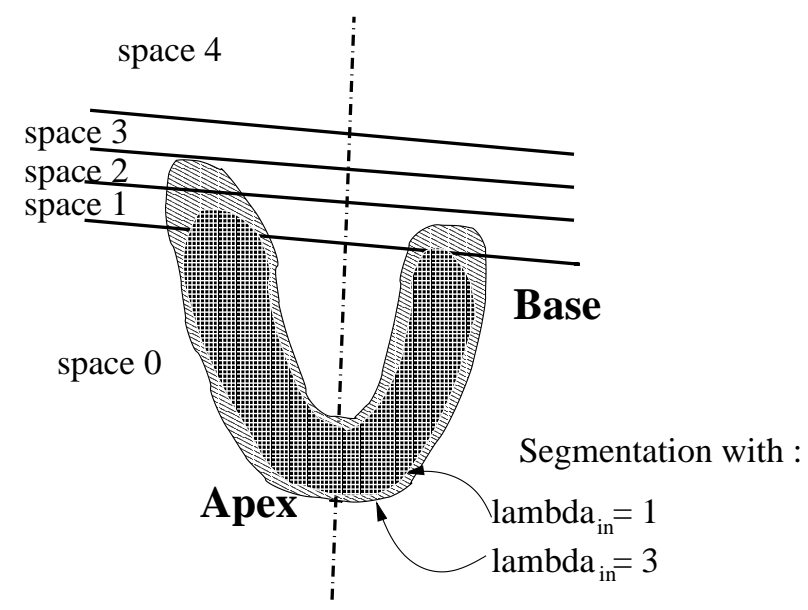

Fig. 6. Splitting the image volume by planes normal to the heart long axis.

Deformation with variable weights. Once the splitting planes have been located, all frames of the cardiac sequence are segmented. Different values are set for the $\lambda_{i n}$ weight in each frame and each volume region. The criterion equation 1 is modified for a new criterion with varying weights $\lambda_{i n}$ :

$$
\frac{\partial u_{n}}{\partial t}=\left(\lambda_{S_{i}, n}\left(I_{n}-\mu_{i n_{S_{i}, n}}\right)-\lambda_{\text {out }}\left(B-I_{n}\right)-\lambda_{c} \kappa_{n}\right)\left\|\nabla u_{n}\right\|
$$

where $S_{i}$ is a $3 \mathrm{D}$ region delimited by planes (see figure 6 ): $S_{0}$ corresponds to the region containing the apex, $S_{4}$ to the region beyond the base.

The different weights were determined empirically on an image dataset. Following the observations made in paragraph 3.1, we choose the $\lambda_{i n}$ weights growing from $S_{0}$ to $S_{4}$ near the diastole, and growing from $S_{4}$ to $S_{0}$ near the systole. The weights used for NCAT simulated sequences are shown in left of figure 7 .

For other sequences, a different weights tuning might be needed. This parameterization is constant for a given image acquisition protocol. Manual tuning is only needed when changing the acquisition device or protocol. 


\begin{tabular}{|l|l|l|l|l|l|l|l|l|}
\hline & 0 & 1 & 2 & 3 & 4 & 5 & 6 & 7 \\
\hline$S_{0}$ & 5 & 5 & 0.5 & 0.2 & 0.2 & 0.2 & 5 & 5 \\
$S_{1}$ & 4 & 4 & 3 & 8 & 8 & 3 & 4 & 4 \\
$S_{2}$ & 2.5 & 2.5 & 3 & 8 & 8 & 3 & 2.5 & 2.5 \\
$S_{3}$ & 1.2 & 1 & 2.5 & 8 & 8 & 2 & 1 & 1.2 \\
$S_{4}$ & 0.4 & 0.6 & 2 & 8 & 8 & 2 & 0.6 & 0.4 \\
\hline
\end{tabular}

\begin{tabular}{|l|l|l|l|l|l|l|l|l|}
\hline & 0 & 1 & 2 & 3 & 4 & 5 & 6 & 7 \\
\hline$S_{0}$ & 3 & 2.5 & 1.5 & 1.5 & 2.5 & 3 & 3 & 3 \\
$S_{1}$ & 2 & 2 & 4 & 4 & 2 & 2 & 2 & 2 \\
$S_{2}$ & 1 & 1.5 & 4 & 4 & 1.5 & 1.5 & 1 & 1 \\
$S_{3}$ & 0.1 & 0.5 & 4 & 4 & 0.5 & 0.12 & 0.2 & 0.1 \\
$S_{4}$ & 0.05 & 0.3 & 4 & 4 & 0.2 & 0.1 & 0.1 & 0.05 \\
\hline
\end{tabular}

Fig. 7. Adaptive weights. On the left, values for NCAT sequences. On the rigth, values used for the real gated SPECT data.

Segmentation results with the adaptive algorithm. The adaptive algorithm segmentation, leads to a good profile for the LV chamber volume variations during the cardiac cycle as shown in figure 8 . The values are close to the real ones with an error of about $15 \mathrm{ml}$ (about 13\%). This is sufficient to compute accurate EF values (with an error of $8 \%$ ).

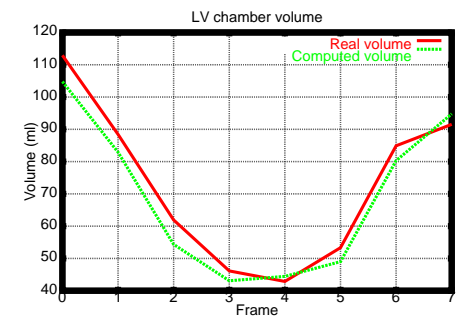

Fig. 8. LV chamber volume variations along the cardiac cycle using NCAT data: real values and computed values.

\subsection{Experiments on real images from healthy patients}

Segmentation experiments were made on real images provided by the Centre Antoine Lacassagne nuclear medicine department in Nice. The images were acquired and filtered by a Butterworth low-pass filter before 3D reconstruction. The voxels dimension is $3.46 \times 3.46 \times 7.12 \mathrm{~mm}$.

Figure 9 shows a segmentation example. The myocardium segmentation (left), the convex envelope extracted by the membrane algorithm (center), and the LV chamber surface (right) are shown for 4 out of the 8 images of a complete sequence.

The weights used for $\lambda_{i n}$ are shown in right of figure 7 . Figure 10 compares the evolution of the LV chamber volume obtained using basic segmentation and the adaptive algorithm. With the later method, the profile of the curve is improved and the $\mathrm{EF}$ value ( $75.5 \%$ instead of $56.5 \%$ ) is more realistic. 


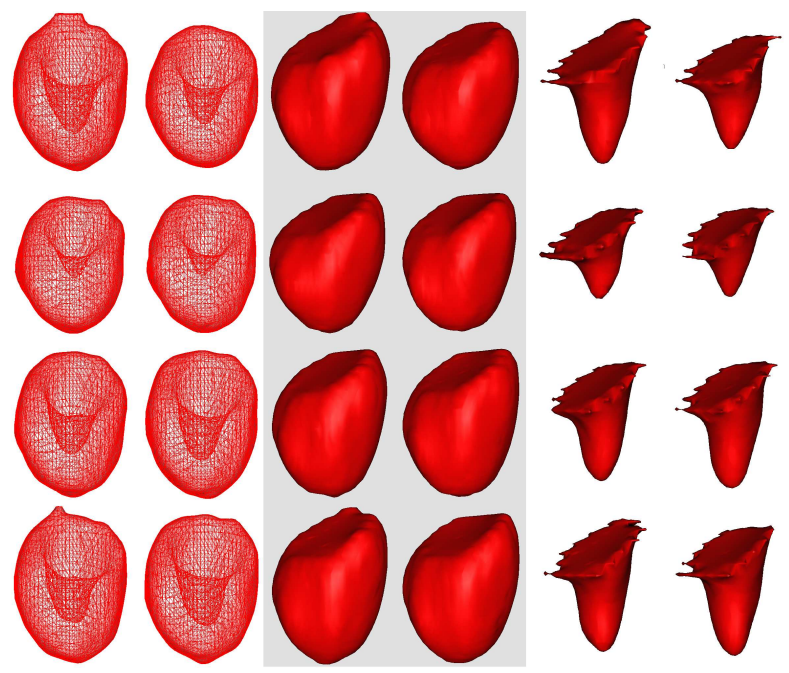

Fig. 9. LV chamber estimation: meshes (computed using Marching Cubes algorithm) of the myocardium segmentation (left), membrane with Gouraud shading (center), and chamber estimation with Gouraud shading (right).

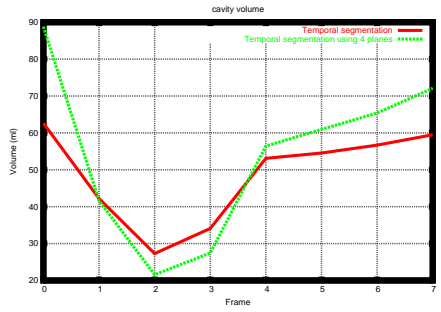

Fig. 10. Comparison of LV chamber volume variations during the cardiac cycle, on real SPECT data, from a healthy patient. 


\section{Conclusion}

The accurate estimation of the LV myocardium and chamber volumes is very sensitive to segmentation errors in gated SPECT images. In this paper, we proposed a novel adaptive algorithm taking into account the temporal nature of the image sequences to more precisely locate the heart wall boundaries. Our algorithm uses the whole image sequence to estimate the background intensity in the deformation criterion 1. Furthermore, the temporal blurring of the sequences is compensated through the spatial and temporal adaptation of the algorithm parameters. A membrane algorithm was developed to automatically extract the LV chamber from the myocardium segmentation.

We could validate the accuracy of the method on simulated images. The error in the LV chamber volume computation do not exceed $15 \mathrm{ml}$. The resulting variability of the EF is about $8 \%$ which is low enough for a practical use. First results on real images are encouraging although a clinical study is needed to compare the results to established gold standards. Setting the adaptive parameters automatically is highly desirable from the user point of view. Some work also need to be done in the case of pathological images showing severe signal drops due to myocardium perfusion defaults: holes then appear in the myocardium wall that need to be taken into account for volumes estimation.

\section{References}

1. I. Buvat, M.L. Bartlett, A.N. Kitsiou, V. Dilsizian, and S.L. Bacharach. A hybrid method for measuring myocardial wall thickening from gated pet/spect images. Journal of Nuclear Medicine, 38(2):324-329, 1997.

2. V. Caselles, F. Catte, T. Coll, and F. Dibos. A geometric model for active contours in image processing. In Numerische Mathematik, volume 66, pages 1-33, 1993.

3. V. Caselles, R. Kimmel, and G. Sapiro. Geodesic active contours. International Journal of Computer Vision, 22(1):61-79, 1997.

4. A. Charnoz, D. Lingrand, and J. Montagnat. A levelset based method for segmenting the heart in $3 \mathrm{~d}+\mathrm{t}$ gated spect images. In Functional Imaging and Modeling of the Heart (FIMH) 2003, LNCS, pages 52-61. Springer-Verlag, June 2003.

5. L.D. Cohen and Ron Kimmel. Global minimum for active contour models: A minimal path approach. Int. J. of Computer Vision, 24(1):57-78, 1997.

6. D. Cremers and S. Soatto. A pseudo distance for shape priors in level set segmentation. In 2nd IEEE International Workshop on Variational, Geometric and Level Set Methods (VLSM), pages 169-176, 2003.

7. E. Debreuve, M. Barlaud, G. Aubert, I. Laurette, and J. Darcourt. Space-Time Segmentation Using Level Set Active Contours Applied to Myocardial Gated SPECT. IEEE Transactions on Medical Imaging, 20(7):643-659, 2001.

8. M. Droske, B. Meyer, M. Rumpf, and C. Schaller. An adaptive level set method for medical image segmentation. In R. Leahy and M. Insana, editors, Proc. of the Annual Symposium on Information Processing in Medical Imaging. Springer, Lecture Notes Computer Science, 2001.

9. Stegmann etal. Fame - a flexible appearance modeling environment. IEEE Trans. on Medical Imaging, 22(10), 2003. 
10. T.L. Faber, C.D. Cooke, R.D. Folks, J.P. Vansant, K.J. Nichos, E.G. DePuey, R.I. Pettigrew, and E.V. Garcia. Left ventricular function and perfusion from gated spect perfusion images : An intergrated method. Journal of Nuclear Medicine, 40:650-659, 1999.

11. J. Gomes and O. Faugeras. Segmentation of the inner and outer surfaces of the cortex in man and monkey: an approach based on Partial Differential Equations. In Proc. of the 5th Int. Conf. on Functional Mapping of the Human Brain, 1999.

12. S. Jehan-Besson, M. Barlaud, and G. Aubert. A 3-step algorithm using regionbased active contours for video objects detection. EURASIP Journal of Applied Signal Processing, 2002(6):572-581, 2002.

13. R. Malladi, J. A. Sethian, and B.C. Vemuri. Shape modeling with front propagation: A level set approach. IEEE Transactions on Pattern Analysis and Machine Intelligence, 17(2):158-175, February 1995.

14. R. Malladi and J.A. Sethian. A Real-Time Algorithm for Medical Shape Recovery. In International Conference on Computer Vision (ICCV'98), pages 304-310, Bombay, India, January 1998.

15. J. Montagnat and H. Delingette. A review of deformable surfaces: topology, geometry and deformation. Image and Vision Comput., 19(14):1023-1040, Dec. 2001.

16. S. Osher and J. Sethian. Fronts propagating with curvature dependent speed : algorithms based on the Hamilton-Jacobi formulation. Journal of Computational Physics, 79:12-49, 1988.

17. Nikos Paragios and Rachid Deriche. Geodesic active contours and level sets for the detection and tracking of moving objects. IEEE Transactions on Pattern Analysis and Machine Intelligence, 22(3):266-280, March 2000.

18. Nikos Paragios, Mikael Rousson, and Visvanathan Ramesh. Knowledge-based registration and segmentation of the left ventricle: A level set approach. In IEEE Workshop on Applications in Computer Vision, Orlando, Florida, December 2002.

19. P.H. Pretorius, W. Xia, M. A. King, B. M. W. Tsui, T.-S. Pan, and B.J. Villegas. Determination of left and right ventricular volume and ejection fraction using a mathematical cardiac torso phantom for gated blood pool spect. Journal of Nuclear Medicine, 37:97, 1996.

20. D. Terzopoulos, A. Witkin, and M. Kass. Constraints on deformable models : Recovering 3d shape and non rigid motion. Artificial Intelligence, 36(1):91-123, 1988. 